\title{
Universal low-temperature behavior of two-dimensional lattice scalar chromodynamics
}

\author{
Claudio Bonati $\odot,{ }^{1}$ Andrea Pelissetto $\odot,{ }^{2}$ and Ettore Vicari ${ }^{1}$ \\ ${ }^{1}$ Dipartimento di Fisica dell'Università di Pisa and INFN Largo Pontecorvo 3, I-56127 Pisa, Italy \\ ${ }^{2}$ Dipartimento di Fisica dell'Università di Roma Sapienza and INFN Sezione di Roma I, \\ I-00185 Roma, Italy
}

(Received 29 January 2020; accepted 24 February 2020; published 11 March 2020)

\begin{abstract}
We study the role that global and local non-Abelian symmetries play in two-dimensional (2D) lattice gauge theories with multicomponent scalar fields. We start from a maximally $\mathrm{O}(M)$-symmetric multicomponent scalar model. Its symmetry is partially gauged to obtain an $\mathrm{SU}\left(N_{c}\right)$ gauge theory (scalar chromodynamics) with global $\mathrm{U}\left(N_{f}\right)$ (for $N_{c} \geq 3$ ) or $\mathrm{Sp}\left(N_{f}\right)$ symmetry (for $N_{c}=2$ ), where $N_{f}>1$ is the number of flavors. Correspondingly, the fields belong to the coset $S^{M} / \mathrm{SU}\left(N_{c}\right)$ where $S^{M}$ is the $M$-dimensional sphere and $M=2 N_{f} N_{c}$. In agreement with the Mermin-Wagner theorem, the system is always disordered at finite temperature and a critical behavior only develops in the zero-temperature limit. Its universal features are investigated by numerical finite-size scaling methods. The results show that the asymptotic low-temperature behavior belongs to the universality class of the $2 \mathrm{D} \mathrm{CP}^{N_{f}-1}$ field theory for $N_{c}>2$ and to that of the $2 \mathrm{D} \mathrm{Sp}\left(N_{f}\right)$ field theory for $N_{c}=2$. These universality classes correspond to $2 \mathrm{D}$ statistical field theories associated with symmetric spaces that are invariant under $\operatorname{Sp}\left(N_{f}\right)$ transformations for $N_{c}=2$ and under $\mathrm{SU}\left(N_{f}\right)$ for $N_{c}>2$. These symmetry groups are the same invariance groups of scalar chromodynamics, apart from a U(1) flavor symmetry that is present for $N_{f} \geq N_{c}>2$, which does not play any role in determining the asymptotic behavior of the model.
\end{abstract}

DOI: $10.1103 /$ PhysRevD.101.054503

\section{INTRODUCTION}

Non-Abelian gauge symmetries are known since long time to describe fundamental interactions [1]. More recently, it has been pointed out that they may also characterize emerging phenomena in condensed-matter physics; see, e.g., Refs. [2-6] and references therein. As a consequence, the large-scale properties of gauge models are also of interest in two or three dimensions.

We consider a lattice model of interacting scalar fields in the presence of non-Abelian gauge symmetries, which may be named scalar chromodynamics or non-Abelian Higgs model. In four space-time dimensions, it represents a paradigmatic example to discuss the non-Abelian Higgs mechanism, which is at the basis of the Standard Model of fundamental interactions. The three-dimensional model may also be relevant in condensed-matter physics, for systems with emerging non-Abelian gauge symmetries. Its phase diagram and its behavior at the finite-temperature

Published by the American Physical Society under the terms of the Creative Commons Attribution 4.0 International license. Further distribution of this work must maintain attribution to the author(s) and the published article's title, journal citation, and DOI. Funded by SCOAP ${ }^{3}$. phase transitions have been investigated in Refs. [7,8]. In this paper, we extend such a study to two-dimensional (2D) systems.

We consider a 2D lattice non-Abelian gauge theory with multicomponent scalar fields. It is defined starting from a maximally $\mathrm{O}(M)$-symmetric multicomponent scalar model. The global symmetry is partially gauged, obtaining a non-Abelian gauge model, in which the fields belong to the coset $S^{M} / \mathrm{SU}\left(N_{c}\right)$, where $M=2 N_{f} N_{c}, N_{f}$ is the number of flavors, and $S^{M}=\mathrm{SO}(M) / \mathrm{SO}(M-1)$ is the $M$-dimensional sphere. According to the Mermin-Wagner theorem [9], the model is always disordered for finite values of the temperature. However, a critical behavior develops in the zero-temperature limit. We investigate its universal features for generic values of $N_{c}$ and $N_{f} \geq 2$, by means of finite-size scaling (FSS) analyses of Monte Carlo (MC) simulations.

The results provide numerical evidence that the asymptotic low-temperature behavior of these lattice non-Abelian gauge models belongs to the universality class of the $2 \mathrm{D}$ $\mathrm{CP}^{N_{f}-1}$ field theory when $N_{c} \geq 3$ and to that of the 2D $\operatorname{Sp}\left(N_{f}\right)$ field theory for $N_{c}=2$. This suggests that the renormalization-group (RG) flow of the 2D multiflavor lattice scalar chromodynamics associated with the coset $S^{M} / \mathrm{SU}\left(N_{c}\right)$ is asymptotically controlled by the $2 \mathrm{D}$ 
statistical field theories associated with the symmetric spaces $[10,11]$ that have the same global symmetry, i.e., $\mathrm{SU}\left(N_{f}\right)$ for $N_{c} \geq 3$ and $\operatorname{Sp}\left(N_{f}\right)$ for $N_{c}=2$.

The paper is organized as follows. In Sec. II, we introduce the lattice non-Abelian gauge models that we consider. In Sec. III, we discuss the general strategy we use to investigate the nature of the low-temperature critical behavior. Then, in Secs. IV and V, we report the numerical results for lattice models with $N_{c} \geq 3$ and $N_{c}=2$, respectively. Finally, in Sec. VI, we summarize and draw our conclusions. In Appendix, we report some results on the minimum-energy configurations of the models considered.

\section{MULTIFLAVOR LATTICE SCALAR CHROMODYNAMICS}

We consider a 2D lattice scalar non-Abelian gauge theory obtained by partially gauging a maximally symmetric model of complex matrix variables $\varphi_{x}^{a f}$, where the indices $a=1, \ldots, N_{c}$ and $f=1, \ldots, N_{f}$ are associated with the color and flavor degrees of freedom, respectively.

We start from the maximally symmetric action

$$
S_{s}=-t \sum_{x, \mu} \operatorname{Re} \operatorname{Tr} \varphi_{x}^{\dagger} \varphi_{x+\hat{\mu}}, \quad \operatorname{Tr} \varphi_{x}^{\dagger} \varphi_{x}=1,
$$

where the sum is over all sites and links of a square lattice and $\hat{\mu}=\hat{1}, \hat{2}$ denote the unit vectors along the lattice directions. Model (1) with the unit-length constraint for the $\varphi_{x}$ variables is a particular limit of a model with a quartic potential $\sum_{x} V\left(\operatorname{Tr} \varphi_{x}^{\dagger} \varphi_{x}\right)$ of the form $V(X)=r X+\frac{1}{2} u X^{2}$. Indeed, it can be obtained by simply setting $r+u=0$ and taking the limit $u \rightarrow \infty$. In the following, we set $t=1$ for simplicity, which amounts to an appropriate choice of the temperature unit. It is simple to see that the action $S_{s}$ has a global $\mathrm{O}(M)$ symmetry, with $M=2 N_{f} N_{c}$. Indeed, it can also be written in terms of $M$-component real vectors $\boldsymbol{s}_{\boldsymbol{x}}$ (which are the real and imaginary parts of $\varphi_{x}^{a f}$ ) as

$$
S_{s}=-\sum_{x, \mu} s_{x} \cdot s_{x+\hat{\mu}}, \quad s_{x} \cdot s_{x}=1 .
$$

This is the standard nearest-neighbor $M$-vector lattice model.

We proceed by gauging some of the degrees of freedom using the Wilson approach [12]. We associate an $\mathrm{SU}\left(N_{c}\right)$ matrix $U_{\boldsymbol{x}, \mu}$ with each lattice link $[(\boldsymbol{x}, \mu)$ denotes the link that starts at site $\boldsymbol{x}$ in the $\hat{\mu}$ direction] and add a Wilson kinetic term for the gauge fields. We obtain the action of the 2D lattice scalar chromodynamics defined by

$$
S_{g}=-N_{f} \sum_{x, \mu} \operatorname{Re} \operatorname{Tr} \varphi_{x}^{\dagger} U_{x, \mu} \varphi_{x+\hat{\mu}}-\frac{\gamma}{N_{c}} \sum_{x} \operatorname{Re} \operatorname{Tr} \Pi_{x},
$$

where $\Pi_{x}$ is the plaquette operator

$$
\Pi_{x}=U_{x, \hat{1}} U_{x+\hat{1}, 2} U_{x+\hat{2}, 1}^{\dagger} U_{x, 2}^{\dagger} .
$$

The plaquette parameter $\gamma$ plays the role of inverse gauge coupling, and the $N_{f}$ and $N_{c}$ factors in Eq. (3) are conventional. The partition function reads

$$
Z=\sum_{\{\varphi, U\}} e^{-\beta S_{g}}, \quad \beta \equiv 1 / T .
$$

The lattice model (3) is invariant under $\mathrm{SU}\left(N_{c}\right)$ gauge transformations,

$$
\varphi_{x} \rightarrow W_{x} \varphi_{x}, \quad U_{x, \mu} \rightarrow W_{x} U_{x, \mu} W_{x+\hat{\mu}}^{\dagger},
$$

with $W_{x} \in \mathrm{SU}\left(N_{c}\right)$. For $\gamma \rightarrow \infty$, the link variables $U_{x}$ become equal to the identity (modulo gauge transformations); thus, one recovers the ungauged model (1), or equivalently the $\mathrm{O}(M)$ vector model (2).

For $N_{f}=1$, the model is trivial. Because of the unitlength condition, using gauge transformations we can fix $\varphi_{x}$ to any given unit-length vector on the whole lattice: there is no dynamics associated with the scalar field. As we shall see, multiflavor models with $N_{f} \geq 2$ show instead a nontrivial behavior.

After gauging, the residual global symmetry depends on the number of flavors $N_{f}$ and of colors $N_{c}$. For $N_{c} \geq 3$, model (3) is invariant under the transformation

$$
\varphi_{x} \rightarrow \varphi_{x} V, \quad V \in \mathrm{U}\left(N_{f}\right),
$$

thus, it has a global $U\left(N_{f}\right) / \mathbb{Z}_{N_{c}}$ symmetry, $\mathbb{Z}_{N_{c}}$ being the center of $\mathrm{SU}\left(N_{c}\right)$. As discussed in Ref. [8], when $N_{f}<N_{c}$,

$$
\varphi_{x} \rightarrow e^{i \theta} \varphi_{x}
$$

can be realized by an appropriate $\mathrm{SU}\left(N_{c}\right)$ local transformation. Thus, the actual global symmetry group reduces to $\mathrm{SU}\left(N_{f}\right)$.

For $N_{c}=2$, model (3) is invariant under the larger group $\operatorname{Sp}\left(N_{f}\right) / \mathbb{Z}_{2}$, where $\operatorname{Sp}\left(N_{f}\right) \supset U\left(N_{f}\right)$ is the compact complex symplectic group; see also Refs. [2,7,8,13,14]. Indeed, if one defines the $2 \times 2 N_{f}$ matrix field,

$$
\Gamma_{\boldsymbol{x}}^{a f}=\varphi_{\boldsymbol{x}}^{a f}, \quad \Gamma_{x}^{a\left(N_{f}+f\right)}=\sum_{b} \epsilon^{a b} \bar{\varphi}_{x}^{b f},
$$

where $f=1, \ldots, N_{f}, \epsilon^{a b}=-\epsilon^{b a}, \epsilon^{12}=1$, the action (3) is invariant under the global transformation

$$
\Gamma_{x}^{a l} \rightarrow \sum_{m=1}^{2 N_{f}} \Gamma_{x}^{a m} Y^{m l}, \quad Y \in \operatorname{Sp}\left(N_{f}\right) .
$$

We recall that the compact complex symplectic group $\mathrm{Sp}\left(N_{f}\right)$ is the group of the $2 N_{f} \times 2 N_{f}$ unitary matrices $U_{\mathrm{sp}}$ satisfying the condition 


$$
U_{\mathrm{sp}} J U_{\mathrm{sp}}^{T}=J, \quad J=\left(\begin{array}{cc}
0 & -I \\
I & 0
\end{array}\right),
$$

where $I$ is the $N_{f} \times N_{f}$ identity matrix.

\section{UNIVERSAL FINITE-SIZE SCALING}

We exploit FSS techniques [15-18] to study the nature of the asymptotic critical behavior of the model for $T \rightarrow 0$. For this purpose, we consider models defined on square lattices of linear size $L$ with periodic boundary conditions.

We mostly focus on the correlations of the gaugeinvariant variable $Q_{x}$ defined by

$$
Q_{x}^{f g}=P_{x}^{f g}-\frac{1}{N_{f}} \delta^{f g}, \quad P_{x}^{f g}=\sum_{a} \bar{\varphi}_{x}^{a f} \varphi_{x}^{a g},
$$

which is a Hermitian and traceless $N_{f} \times N_{f}$ matrix. The corresponding two-point correlation function is defined as

$$
G(\boldsymbol{x}-\boldsymbol{y})=\left\langle\operatorname{Tr} Q_{x} Q_{\boldsymbol{y}}\right\rangle,
$$

where the translation invariance of the system has been taken into account. We define the susceptibility $\chi=\sum_{x} G(\boldsymbol{x})$ and the correlation length

$$
\xi^{2}=\frac{1}{4 \sin ^{2}(\pi / L)} \frac{\tilde{G}(\mathbf{0})-\tilde{G}\left(\boldsymbol{p}_{m}\right)}{\tilde{G}\left(\boldsymbol{p}_{m}\right)},
$$

where $\tilde{G}(\boldsymbol{p})=\sum_{x} e^{i \boldsymbol{p} \cdot \boldsymbol{x}} G(\boldsymbol{x})$ is the Fourier transform of $G(\boldsymbol{x})$ and $\boldsymbol{p}_{m}=(2 \pi / L, 0)$. We also consider the quartic cumulant (Binder) parameter defined as

$$
U=\frac{\left\langle\mu_{2}^{2}\right\rangle}{\left\langle\mu_{2}\right\rangle^{2}}, \quad \mu_{2}=\frac{1}{V^{2}} \sum_{x, \boldsymbol{y}} \operatorname{Tr} Q_{x} Q_{y},
$$

where $V=L^{2}$.

To identify the universality class of the asymptotic zerotemperature behavior, we consider the Binder parameter $U$ as a function of the ratio

$$
R_{\xi} \equiv \xi / L .
$$

Indeed, in the FSS limit, we have (see, e.g., Ref. [19])

$$
U(\beta, L) \approx F\left(R_{\xi}\right),
$$

where $F(x)$ is a universal scaling function that completely characterizes the universality class of the transition. Equation (17) is particularly convenient, as it allows us to check the universality of the asymptotic zero-temperature behavior without the need of tuning any parameter. Corrections to Eq. (17) decay as a power of $L$. In the case of asymptotically free models, such as the $2 \mathrm{D} \mathrm{CP}^{N-1}$ and
$\mathrm{O}(N)$ vector models, corrections decrease as $L^{-2}$, multiplied by powers of $\ln L[19,20]$.

Because of the universality of relation (17), we can use the plots of $U$ versus $R_{\xi}$ to identify the models that belong to the same universality class. If the data of $U$ for two different models follow the same curve when plotted versus $R_{\xi}$, their critical behavior is described by the same continuum quantum field theory. This implies that any other dimensionless RG invariant quantity has the same critical behavior in the two models, both in the thermodynamic and in the FSS limit. An analogous strategy was employed in Ref. [19] to study the critical behavior of the 2D Abelian-Higgs lattice model in the zero-temperature limit.

The asymptotic values of $F\left(R_{\xi}\right)$ for $R_{\xi} \rightarrow 0$ and $R_{\xi} \rightarrow \infty$ correspond to the values that $U$ takes in the small- $\beta$ and large- $\beta$ limits. For $R_{\xi} \rightarrow 0$, we have

$$
\lim _{R_{\xi} \rightarrow 0} U=\frac{N_{f}^{2}+1}{N_{f}^{2}-1},
$$

independently of the value of $N_{c}$. The large- $\beta$ limit is discussed in Appendix. For $N_{c} \geq 3$, we have simply $U=1$.

In the following, we study the large- $\beta$ critical behavior of lattice scalar chromodynamics for several values of $N_{f}$ and $N_{c}$. We perform numerical simulations using the same upgrading algorithm employed in three dimensions $[7,8]$. The analysis of the data of $U$ versus $R_{\xi}$ outlined above allows us to conclude that the critical behavior only depends on the global symmetry group of the model. For any $N_{c} \geq 3$, the critical behavior belongs to the universality class of the $2 \mathrm{D} \mathrm{CP} \mathrm{CP}^{N_{f}-1}$ field theory. Indeed, the FSS curves (17) for the model (3) agree with those computed in the $\mathrm{CP}^{N-1}$ model (we use the results reported in Ref. [19]). For $N_{c}=2$, instead, the critical behavior is associated with that of the $2 \mathrm{D} \operatorname{Sp}\left(N_{f}\right)$ field theory. Note that the parameter $\gamma$ appears to be irrelevant in the RG sense (at least for $|\gamma|$ not too large). Indeed, for all positive and negative values of $N_{c}, N_{f}$, and $\gamma$ we investigated, the universal critical behavior does not depend on $\gamma$.

\section{SU( $\left.\boldsymbol{N}_{\boldsymbol{c}}\right)$ GAUGE MODELS WITH $\boldsymbol{N}_{\boldsymbol{c}} \geq 3$}

\section{A. Numerical results}

In this section, we study the critical behavior of scalar chromodynamics for some values of $N_{f}$ and of $N_{c} \geq 3$. We compute the scaling curve (17) and compare it with the corresponding one computed in the $\mathrm{CP}^{N_{f}-1}$ model. Such a comparison provides evidence that the asymptotic zerotemperature behavior for finite values of $\gamma$ in a wide interval around $\gamma=0$ is described by the $2 \mathrm{D} \mathrm{CP}^{N_{f}-1}$ field theory.

In Figs. 1 and 2, we show MC data for the two-flavor model (3) with SU(3) gauge symmetry, i.e., for $N_{f}=2$ and $N_{c}=3$, and $\gamma=0$. In Fig. 1, the results for the Binder 


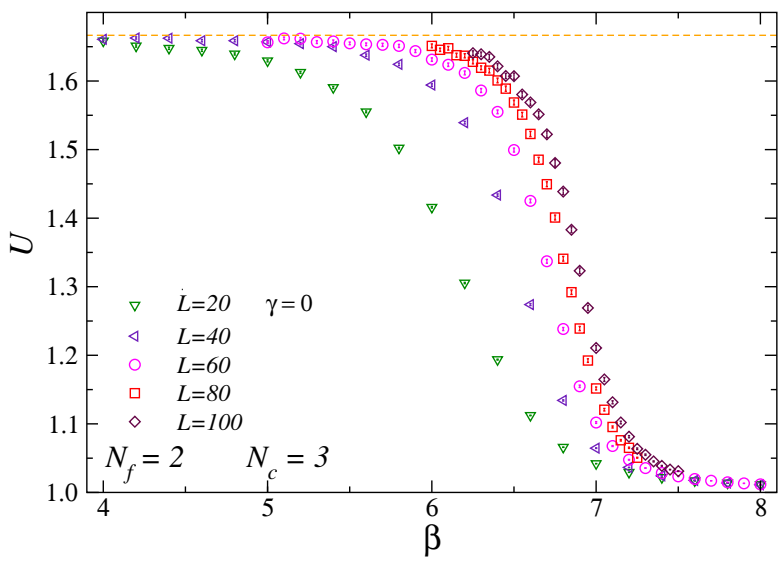

FIG. 1. Plot of $U$ versus $\beta$ for $N_{f}=2, N_{c}=3$, and $\gamma=0$. The horizontal dashed line corresponds to $U=5 / 3$, the asymptotic value for $\beta \rightarrow 0$.

parameter are shown as a function of $\beta$ for several lattice sizes. The curves corresponding to different lattice sizes do not intersect, confirming the absence of a phase transition at finite $\beta$, as expected from the Mermin-Wagner theorem. The ratio $R_{\xi}$ behaves analogously. For each lattice size, $R_{\xi}$ is an increasing function of $\beta$, seemingly divergent for $\beta \rightarrow \infty$, but no crossing is present between curves corresponding to different $L$ values. In Fig. 2, the data of $U$ appear to approach a FSS curve in the large- $L$ limit when plotted versus $R_{\xi}$, in agreement with the FSS prediction (17). This asymptotic FSS curve is consistent with that of the $2 \mathrm{D} \mathrm{CP}^{1}$ universality class (equivalent to that of the $\mathrm{O}(3)$ vector model, see, e.g., Ref. [11]) determined in Ref. [19]. Moreover, scaling corrections are consistent with the expected $O\left(L^{-2}\right)$ behavior.

The behavior of the data for different values of the inverse gauge coupling $\gamma$ shows that the FSS curve is

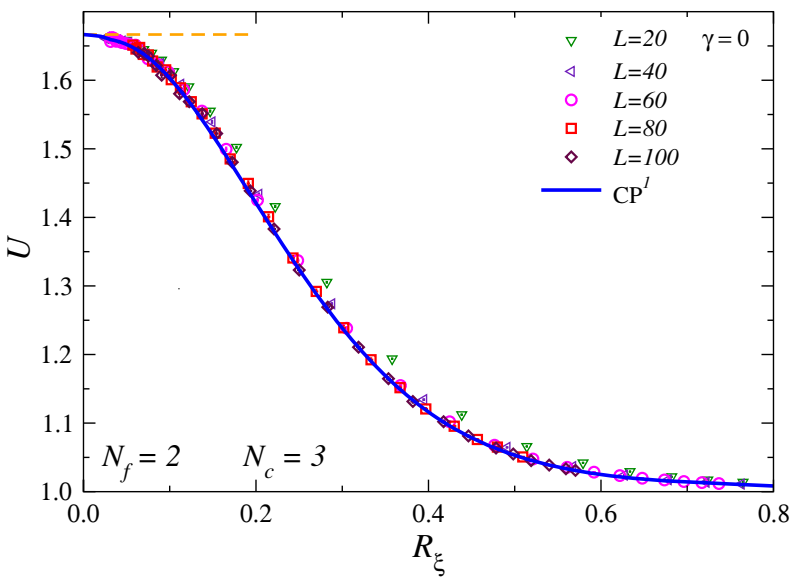

FIG. 2. Plot of $U$ versus $R_{\xi}$ for $N_{f}=2, N_{c}=3$, and $\gamma=0$. Data approach the universal FSS curve of the $2 \mathrm{D} \mathrm{CP}^{1}$ or $\mathrm{O}(3)$ universality class (full line, taken from Ref. [19]). The horizontal dashed line corresponds to $U=5 / 3$, the asymptotic value for $R_{\xi} \rightarrow 0$.
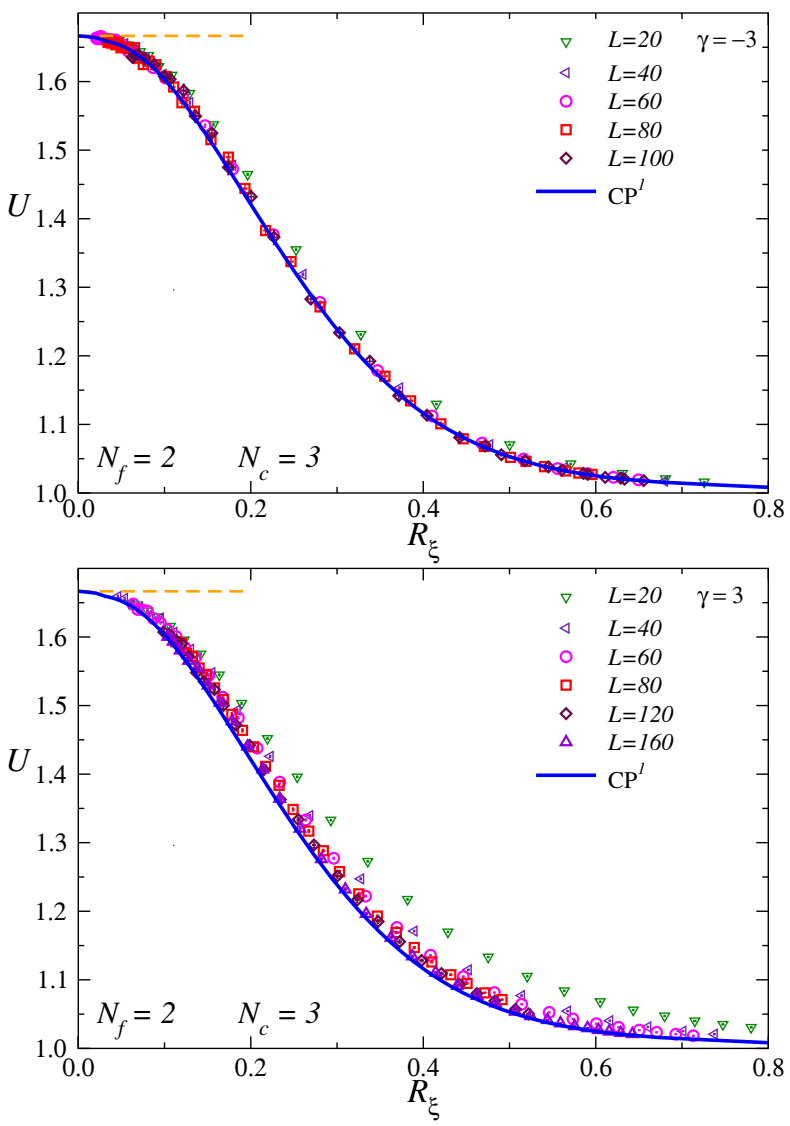

FIG. 3. Plot of $U$ versus $R_{\xi}$ for $N_{f}=2, N_{c}=3$, and $\gamma=-3$ (upper panel) and $\gamma=3$ (lower panel). Data approach the universal FSS curve of the 2D $\mathrm{CP}^{1}$ or $\mathrm{O}(3)$ universality class (full line, taken from Ref. [19]). The horizontal dashed line corresponds to $U=5 / 3$, the asymptotic value for $R_{\xi} \rightarrow 0$.

independent of $\gamma$, at least in a wide interval around $\gamma=0$, as can be seen from Fig. 3, where data for $\gamma= \pm 3$ are reported. Analogous results are obtained for $N_{f}=2$ and $N_{c}=4$; see Fig. 4.

These results should be considered as a robust evidence that the asymptotic low-temperature behavior of two-flavor chromodynamics with $\mathrm{SU}(3)$ and $\mathrm{SU}(4)$ gauge symmetry belongs to the universality class of the $2 \mathrm{D} \mathrm{CP}^{1}$ [equivalently, $\mathrm{O}(3)$ ] field theory.

In Fig. 5, we report results for the three-flavor lattice theory with $\mathrm{SU}(3)$ gauge symmetry. In this case, for both $\gamma=0$ and $\gamma=2$, data appear to approach the FSS curve of the 2D CP ${ }^{2}$ model, obtained in Ref. [19], by numerical simulations. This excellent agreement provides a robust indication that the three-flavor lattice theory with SU(3) gauge group has the same asymptotic critical behavior as the $2 \mathrm{D} \mathrm{CP} \mathrm{CP}^{2}$ model. Analogous results are obtained for $N_{f}=4$; see Fig. 6 for results for $\gamma=0$. The FSS curve appears to approach that of the $2 \mathrm{D} \mathrm{CP}^{3}$ model. We note that for $N_{f}=4$ larger scaling corrections are present. However, they appear to be consistent with an $O\left(L^{-2}\right)$ behavior. 


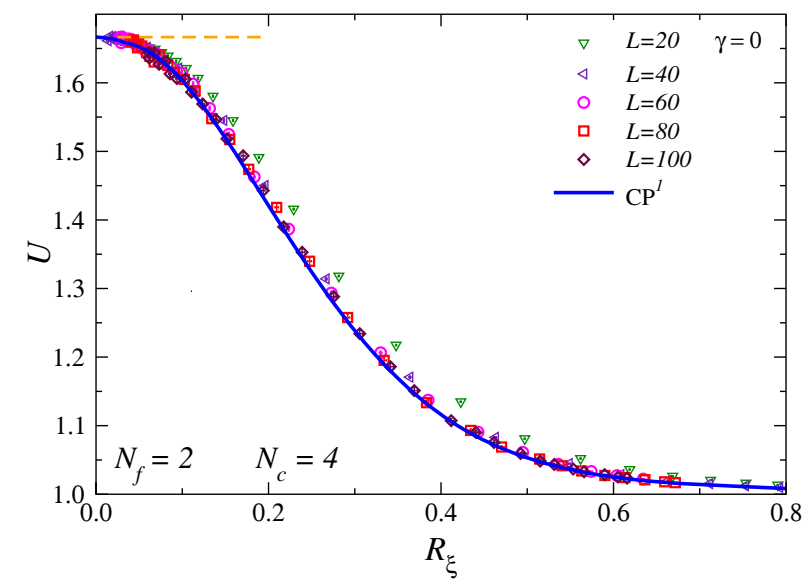

FIG. 4. Plot of $U$ versus $R_{\xi}$ for $N_{f}=2, N_{c}=4$, and $\gamma=0$. Data approach the universal FSS curve of the $2 \mathrm{D} \mathrm{CP}^{1}$ or $\mathrm{O}(3)$ universality class (full line, taken from Ref. [19]). The horizontal dashed line corresponds to $U=5 / 3$, the asymptotic value for $R_{\xi} \rightarrow 0$.

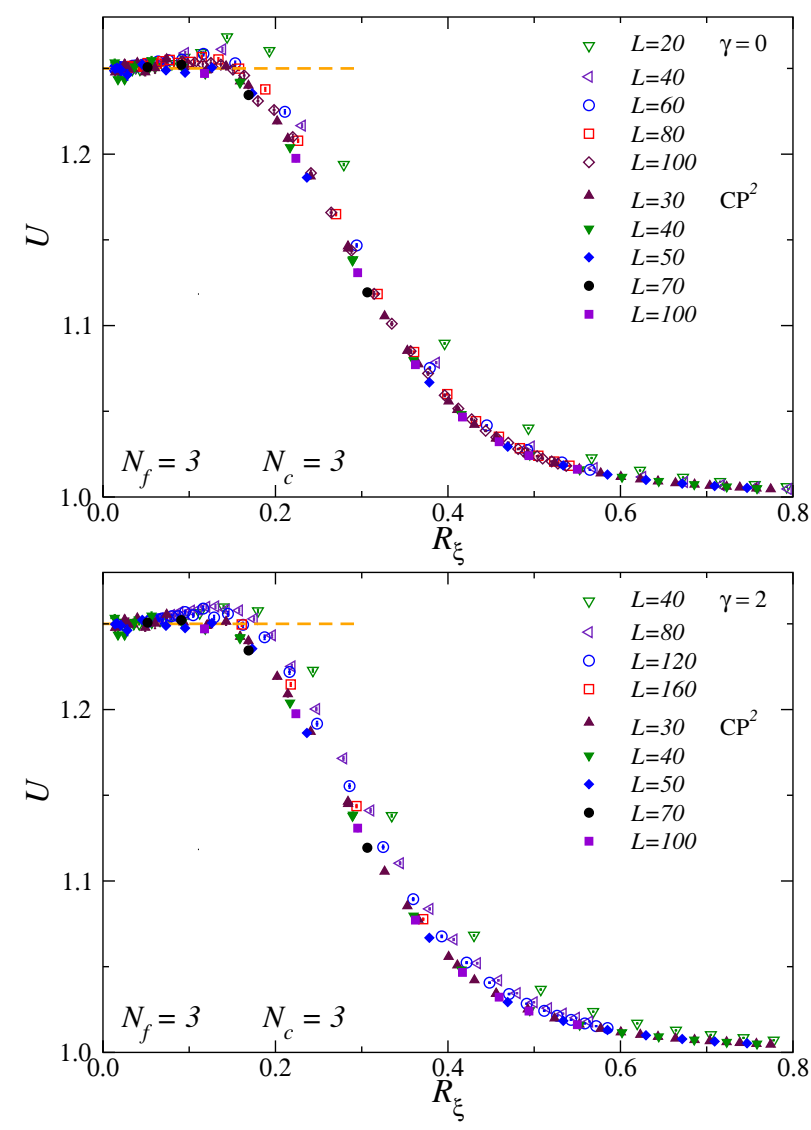

FIG. 5. Plot of $U$ versus $R_{\xi}$ for $N_{f}=3, N_{c}=3$. Results for $\gamma=0$ (top) and $\gamma=2$ (bottom). Data (empty symbols) approach the universal FSS curve of the $2 \mathrm{D} \mathrm{CP} \mathrm{CP}^{2}$ universality class $\left(\mathrm{CP}^{2}\right.$ results, taken from Ref. [19], are reported with full symbols). The horizontal dashed line corresponds to $U=5 / 4$, the asymptotic value for $R_{\xi} \rightarrow 0$.

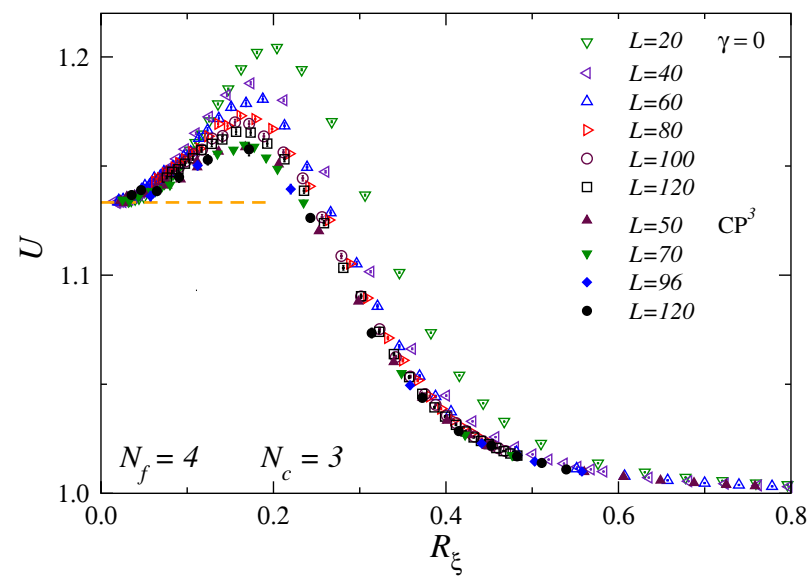

FIG. 6. Plot of $U$ versus $R_{\xi}$ for $N_{f}=4, N_{c}=3$, and $\gamma=0$. Data (empty symbols) approach the universal FSS curve of the 2D CP${ }^{3}$ universality class $\left(\mathrm{CP}^{2}\right.$ results, taken from Ref. [19], are reported with full symbols). The horizontal dashed line corresponds to $U=17 / 15$, the asymptotic value for $R_{\xi} \rightarrow 0$.

Up to now we have discussed the critical behavior of $Q$ correlations. However, note that the model has the additional U(1) global invariance, Eq. (8). As we have already discussed, for $N_{f}<N_{c}$ such an invariance is only apparent, but in principle it may be relevant for $N_{f} \geq N_{c}$. To understand its role, we have studied the behavior of an appropriate order parameter. As discussed in Ref. [8], for $N_{f}=N_{c}$, an order parameter is provided by the composite operator

$$
Y_{x}=\operatorname{det} \varphi_{x}
$$

which is invariant under both the $\mathrm{SU}\left(N_{c}\right)$ gauge transformations (6) and the global transformations $\varphi_{x} \rightarrow \varphi_{x} V$ with $V \in \mathrm{SU}\left(N_{f}\right)$. Starting from $Y_{x}$, one can define a correlation function

$$
G_{Y}(\boldsymbol{x}-\boldsymbol{y})=\left\langle\bar{Y}_{\boldsymbol{x}} Y_{\boldsymbol{y}}\right\rangle
$$

and a correlation length $\xi_{Y}$, using Eq. (14). Results for $\xi_{Y}$ for $N_{f}=N_{c}=3$ and $\gamma=0$ are presented in Fig. 7 . Apparently, $\xi_{Y}$ remain finite and very small $\left(\xi_{Y} \approx 0.5\right)$ as $\beta$ increases. The $\mathrm{U}(1)$ flavor modes are clearly not relevant for the critical behavior, which is completely controlled by the U(1)-invariant modes encoded in $Q_{x}$. The possibility of a U(1) critical behavior, which would imply the presence of a finite-temperature Berezinskii-Kosterlitz-Thouless transition [21-24], is excluded by the MC data. The behavior we observe is completely analogous to what occurs in three dimensions at finite temperature [8].

\section{B. Universality class of the asymptotic low-temperature behavior}

The numerical FSS analyses reported above suggest that, for $N_{c} \geq 3$, the low-temperature asymptotic behavior of 


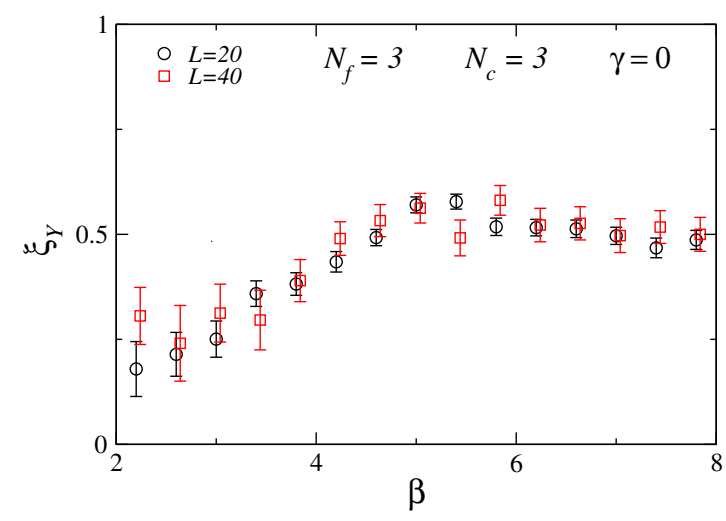

FIG. 7. Plot of the correlation length $\xi_{Y}$ associated with the correlation function $\left\langle\bar{Y}_{x} Y_{y}\right\rangle$ versus $\beta$. The quantity $Y_{x}$ is defined in Eq. (19). Results for $N_{f}=N_{c}=3$ and $\gamma=0$.

scalar chromodynamics with $N_{f}$ flavors depends only on $N_{f}$. Irrespective of the values of $N_{c}$ and of $\gamma$, the critical behavior is the same as that of the $2 \mathrm{D} \mathrm{CP}{ }^{N_{f}-1}$ model.

Before presenting further arguments to support such a conclusion, we recall some features of the $2 \mathrm{D} \mathrm{CP}^{N-1}$ model $[11,25]$. This is a $2 \mathrm{D}$ quantum field theory defined on a complex projective space, isomorphic to the symmetric space $U(N) /[\mathrm{U}(1) \times \mathrm{U}(N-1)]$. Its Lagrangian reads

$$
\begin{aligned}
\mathcal{L} & =\frac{1}{2 g} \overline{D_{\mu} z} \cdot D_{\mu} z, \quad \bar{z} \cdot z=1, \\
D_{\mu} & =\partial_{\mu}+i A_{\mu}, \quad A_{\mu}=i \bar{z} \cdot \partial_{\mu} z,
\end{aligned}
$$

where $z$ is an $N$-component complex field, and $A_{\mu}$ is a composite gauge field. The Lagrangian in invariant under the local U(1) gauge transformations $\boldsymbol{z}(\boldsymbol{x}) \rightarrow e^{i \theta(\boldsymbol{x})} \boldsymbol{z}(\boldsymbol{x})$ and the global transformations $z(x) \rightarrow W z(x)$ with $W \in \mathrm{SU}(N)$. The global invariance group is $\mathrm{SU}(N) / \mathbb{Z}_{N}$ (again global transformations differing by a $\mathbb{Z}_{N}$ factor are gauge equivalent).

For $N=2$, the $\mathrm{CP}^{1}$ field theory is locally isomorphic to the $\mathrm{O}(3)$ nonlinear $\sigma$ model with the identification of the three-component real vector $s_{x}^{a}=\sum_{i j} \bar{z}_{x}^{i} \sigma_{i j}^{a} z_{x}^{j}$, where $a=1,2,3$ and $\sigma^{a}$ are the Pauli matrices. Various lattice formulations of $\mathrm{CP}^{N-1}$ models have been considered; see, e.g., Refs. [26,27]. The simplest formulation is

$$
S_{C P}=-J \sum_{x \mu}\left|\bar{z}_{x} \cdot z_{x+\hat{\mu}}\right|^{2}=-J \sum_{x \mu} \operatorname{Tr} \mathcal{P}_{x} \mathcal{P}_{x+\hat{\mu}},
$$

where

$$
\mathcal{P}_{x}^{a b}=\bar{z}_{x}^{a} z_{x}^{b}
$$

is a projector, i.e., it satisfies $\mathcal{P}_{\boldsymbol{x}}=\mathcal{P}_{\boldsymbol{x}}^{2}$. This explicitly shows that $2 \mathrm{D} \mathrm{CP}{ }^{N-1}$ theories describe the dynamics of projectors on $N$-dimensional complex spaces. $\mathrm{CP}^{N-1}$ models can also be obtained by considering the action (3) with $\gamma=0, z$ replacing the field $\varphi$ and using $\mathrm{U}(1)$ gauge fields $U_{\boldsymbol{x}, \mu}$.

In $2 \mathrm{D} \mathrm{CP}^{N-1}$ models, correlations are always shortranged at finite $\beta$ [9]. A critical behavior is only observed for $T \rightarrow 0$. In this limit, the correlation length increases exponentially as $[11,26]$

$$
\xi \sim T^{p} e^{c / T} .
$$

This behavior is related to the asymptotic-freedom property of these models, which is shared with quantum chromodynamics, the four-dimensional theory of strong interactions. An analogous exponential behavior is expected to characterize all statistical lattice theories belonging to the same universality class, therefore also the $2 \mathrm{D} N$-component Abelian-Higgs lattice model [19] (which is a lattice version of scalar electrodynamics), and, as we shall argue, the 2D scalar chromodynamics with $N_{f}$ flavors and $N_{c} \geq 3$.

The numerical results reported in Sec. IVA show that the asymptotic low-temperature behavior of the model with $N_{c} \geq 3$ is the same as that of the $2 \mathrm{D} \mathrm{CP}{ }^{N_{f}-1}$ models or, equivalently, of the $2 \mathrm{D} N_{f}$-component Abelian-Higgs model with a U(1) gauge symmetry. This is certainly quite surprising. We shall now argue that the correspondence is strictly related to the identical nature of the minimumenergy configurations, which represent the background for the spin waves that are responsible for the zero-temperature critical behavior.

The nature of the minimum-energy configurations is discussed in Appendix. For $\gamma \geq 0$, such configurations are those for which

$$
\operatorname{Re} \operatorname{Tr} \varphi_{x}^{\dagger} U_{x, \mu} \varphi_{x+\hat{\mu}}=1
$$

on each lattice link. In the Appendix, by combining exact and numerical results, we show that, for $\beta \rightarrow \infty$ and $N_{c} \geq 3$, by appropriately fixing the gauge, the configurations that dominate the statistical average have the form

$$
\Pi_{x}=\left(\begin{array}{ll}
V & 0 \\
0 & 1
\end{array}\right)
$$

where $V$ is an $\mathrm{SU}\left(N_{c}-1\right)$ matrix, and

$$
\begin{aligned}
& \varphi^{a f}=0 \quad a<N_{c}, \\
& \varphi^{a f}=z^{f} \quad a=N_{c},
\end{aligned}
$$

where $z^{f}$ is a unit-length $N_{f}$-dimensional vector. In other words, the analysis shows that gauge and $\varphi$ fields completely decouple. Moreover, the $\varphi$ field becomes equivalent to a single unit-length $N_{f}$-dimensional vector, which is the fundamental field of the $\mathrm{CP}^{N_{f}-1}$ model. Stated differently, the operator $P_{x}$ becomes a projector, i.e., satisfies $P_{x}^{2}=P_{x}$, 
for $T \rightarrow 0$. However, we cannot yet, at this point, argue that the large- $\beta$ behavior of scalar chromodynamics and of the $\mathrm{CP}^{N_{f}-1}$ model is the same, because in our factorization there is no U(1) gauge symmetry. However, our numerical data also show that the critical behavior is only associated with the order parameter $Q_{x}$ : the $\mathrm{U}(1)$ modes do not order in the large- $\beta$ limit. This is also confirmed by the detailed analysis of the low-temperature configurations presented in Ref. [8]. Therefore, in the effective theory, we can quotient out the U(1) degrees of freedom, which are irrelevant for the behavior of the order parameter $Q_{x}$, i.e., we can reintroduce the $\mathrm{U}(1)$ gauge symmetry. If this occurs, scalar chromodynamics and $\mathrm{CP}^{N_{f}-1}$ model are expected to have the same critical large- $\beta$ behavior.

It is interesting to observe that $\mathrm{CP}^{N_{f}-1}$ behavior has also been observed for several negative values of $\gamma$. This is not an obvious result, as the system is frustrated. Also, in this case, the result is explained by the nature of the lowtemperature configurations. As discussed in Appendix for a specific value of $\gamma, \gamma=-1$, the relevant configurations can again be parametrized as in Eq. (27), modulo gauge transformations.

This phenomenological argument explains the numerical evidence that the asymptotic zero-temperature behaviors for $N_{c} \geq 3$ is the same as that of the $\mathrm{CP}^{N_{f}-1}$ continuum theory. Note that this scenario does not apply to nonAbelian gauge theories with $N_{c}=2$. As discussed in Appendix, the typical low-temperature configurations cannot be parametrized as in Eq. (27), implying a different critical behavior. We shall argue that it corresponds to that of the $2 \mathrm{D} \operatorname{Sp}\left(N_{f}\right)$ field theories.

\section{SU(2) GAUGE MODELS}

We now discuss the behavior of models with SU(2) gauge symmetry. In this case, the global symmetry group $[7,8]$ is $\mathrm{Sp}\left(N_{f}\right) / \mathbb{Z}_{2}$. In the two-flavor case, because of the isomorphism $\mathrm{Sp}(2) / \mathbb{Z}_{2}=\mathrm{SO}(5)$, an $\mathrm{O}(5)$ symmetry emerges. Because of the symmetry enlargement, the orderparameter field is the $2 N_{f} \times 2 N_{f}$ matrix,

$$
\mathcal{T}_{x}^{l m}=\sum_{a} \bar{\Gamma}_{x}^{a l} \Gamma_{x}^{a m}-\frac{\delta^{l m}}{N_{f}},
$$

where the matrix $\Gamma_{x}$ is defined in Eq. (9). If $f, g=$ $1, \ldots, N_{f}, \mathcal{T}_{x}^{l m}$ can be written in the block form

$$
\begin{aligned}
\mathcal{T}_{x}^{f, g} & =Q_{x}^{f g} & \mathcal{T}_{x}^{f, g+N_{f}}=\bar{D}^{f g} \\
\mathcal{T}_{x}^{f+N_{f}, g} & =-D^{f g} & \mathcal{T}_{x}^{f+N_{f}, g+N_{f}}=Q_{x}^{g f},
\end{aligned}
$$

where

$$
D_{x}^{f g}=\sum_{a b} \epsilon^{a b} \varphi_{x}^{a f} \varphi_{x}^{b g}
$$

The order parameter $\mathcal{T}_{\boldsymbol{x}}$ is Hermitian and satisfies

$$
J \overline{\mathcal{T}}_{x} J+\mathcal{T}_{x}=0,
$$

where the matrix $J$ is defined in Eq. (11).

For $N_{f}=2$, the matrix $\mathcal{T}_{x}$ can be parametrized by a fivedimensional real vector $\boldsymbol{\Phi}_{x}$. The first three components are given by

$$
\Phi_{x}^{k} \equiv \sum_{f g} \sigma_{f g}^{k} Q_{x}^{f g}, \quad k=1,2,3
$$

while the fourth and fifth components are the real and imaginary parts of

$$
\frac{1}{2} \sum_{f g} \epsilon_{f g} D_{x}^{f g} \equiv \Phi_{x}^{4}+i \Phi_{x}^{5}
$$

The parametrization of $\mathcal{T}_{x}$ in terms of $\boldsymbol{\Phi}_{x}$ effectively implements the isomorphism between the $\mathrm{Sp}(2) / \mathbb{Z}_{2}$ and the $\mathrm{SO}(5)$ groups, since an $\mathrm{Sp}(2)$ transformation of $\mathcal{T}_{\boldsymbol{x}}$ maps to an $\mathrm{SO}(5)$ rotation of $\boldsymbol{\Phi}_{x}$. Moreover, the unit-length condition for $\varphi$ implies

$$
\boldsymbol{\Phi}_{x} \cdot \boldsymbol{\Phi}_{x}=1
$$

The discussion of the previous section leads us to conjecture that the global $\operatorname{Sp}\left(N_{f}\right) / \mathbb{Z}_{2}$ symmetry uniquely determines the asymptotic zero-temperature critical behavior. For $N_{f}=2$, this would imply that the $\mathrm{SU}(2)$ gauge theory has the same zero-temperature behavior of the $\mathrm{O}(5)$ vector model. An analogous conjecture proved to be true in the three-dimensional case $[7,8]$. To perform the correct universality check for $N_{f}=2$, as discussed in detail in Ref. [8], it is important to consider a Binder parameter in the $\mathrm{SU}(2)$ gauge theory that maps onto the usual vector $\mathrm{O}(5)$ Binder parameter under the isomorphism $\mathrm{Sp}\left(N_{2}\right) / \mathbb{Z}_{2} \rightarrow \mathrm{SO}(5)$. The Binder parameter $U$ defined in Eq. (15) is not the appropriate one since it only involves three components of $\Phi_{x}^{k}$; see Eq. (32). A straightforward group-theory computation shows that the correct correspondence is achieved by defining the related quantity $[7,8]$

$$
U_{r}=\frac{21}{25} U .
$$

As for $R_{\xi}$, the quantity computed using Eq. (14) corresponds exactly to the analogous quantity computed in the $\mathrm{O}(5)$ vector model.

The results shown in Fig. 8 clearly support the conjecture. Indeed, the $\mathrm{MC}$ data of $U_{r}$ collapse (without appreciable scaling violations) on a unique curve when plotted versus $R_{\xi}$, which is consistent with that of the Binder parameter $U$ versus $R_{\xi}$ for the $2 \mathrm{D} \mathrm{O}(5)$ vector model (with $U$ and $R_{\xi}$ defined analogously in terms of 


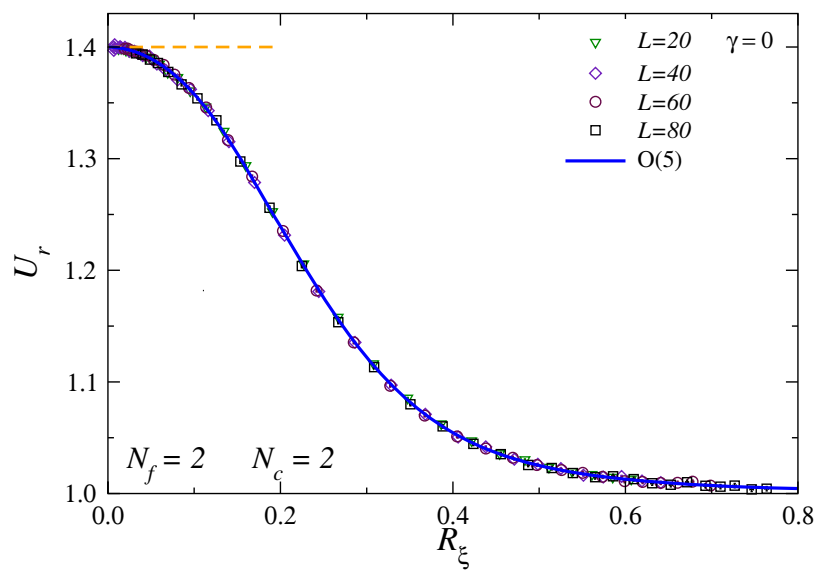

FIG. 8. Plot of $U_{r}$ versus $R_{\xi}$ for $N_{f}=2, N_{c}=2$, and $\gamma=0$. The data approach a universal FSS curve, which corresponds to that of the standard $\mathrm{O}(5)$ nearest-neighbor vector model (full line, see Fig. 9). The horizontal dashed line corresponds to the asymptotic value $U_{r}=7 / 5$ for $R_{\xi} \rightarrow 0$.

$\Phi$ correlations [19]). The O(5) FSS curve is obtained by MC simulations (using the cluster algorithm) of the nearestneighbor $\mathrm{O}(5)$ vector model (2); see Fig. 9. Again the role of the inverse gauge coupling is irrelevant. It does not change the universal features of the low-temperature asymptotic behavior, as shown in Fig. 10, where we report results for $\gamma=2$ and -2 .

The numerical analysis reported above leads us to conjecture that the low-temperature asymptotic behavior of the scalar $\mathrm{SU}(2)$ chromodynamics with $N_{f}$ flavors belongs to the universality class associated with the 2D $\operatorname{Sp}\left(N_{f}\right)$ field theory. The fundamental field is a complex

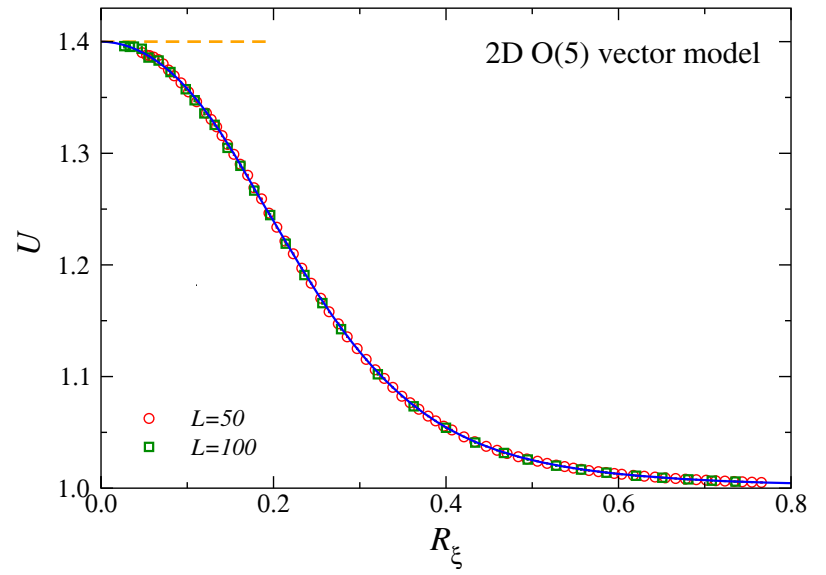

FIG. 9. Plot of $U$ versus $R_{\xi}$ for the $\mathrm{O}(5)$ vector universality class, as obtained by MC simulations of the nearest-neighbor $\mathrm{O}(5)$ vector lattice model. The full line [28] is an interpolation the MC data up to $R_{\xi} \lesssim 0.8$. It provides an approximation of the universal FSS curve, with an accuracy smaller than $0.5 \%$ (we include the uncertainty arising from scaling corrections). The horizontal dashed line corresponds to the asymptotic value $U=7 / 5$ for $R_{\xi} \rightarrow 0 ; U \rightarrow 1$ for $R_{\xi} \rightarrow \infty$.

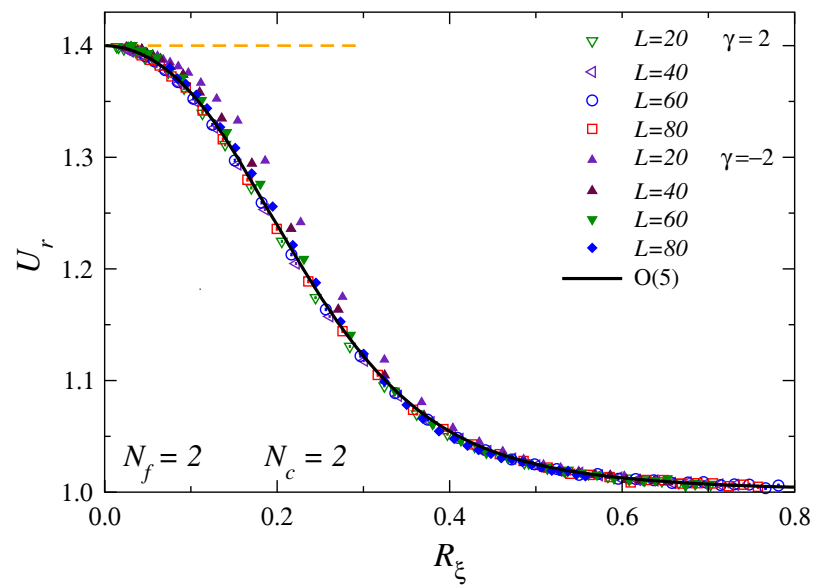

FIG. 10. Plot of $U_{r}$ versus $R_{\xi}$ for $N_{f}=2, N_{c}=2$, and $\gamma= \pm 2$. The data approach the O(5) FSS curve in the large $L$ limit (full line [28]). The horizontal dashed line corresponds to the asymptotic value $U_{r}=7 / 5$ for $R_{\xi} \rightarrow 0$.

$2 N_{f} \times 2 N_{f}$ order-parameter field $\Psi_{x}$, which formally represents a coarse-grained version of $\mathcal{T}_{x}$, defined in Eq. (28). It is Hermitian, traceless, and satisfies Eq. (31). If we write

$$
\Psi=\left(\begin{array}{ll}
A_{1} & A_{2} \\
A_{3} & A_{4}
\end{array}\right)
$$

where $A_{i}$ are $N_{f} \times N_{f}$ matrix fields, the conditions required are that $A_{1}$ is Hermitian and traceless, $A_{3}$ is antisymmetric, $A_{4}=\bar{A}_{1}$, and $A_{3}=-\bar{A}_{2}$. The corresponding $2 \mathrm{D}$ field theory is defined by the Lagrangian,

$$
\mathcal{L}_{\mathrm{Sp}}=\frac{1}{g} \operatorname{Tr}\left[\partial_{\mu} \Psi^{\dagger} \partial_{\mu} \Psi\right], \quad \operatorname{Tr} \Psi \dagger \Psi=1 .
$$

For $N_{f}=2$, using the correspondence

$$
\begin{aligned}
& A_{1}=\frac{1}{2}\left(\begin{array}{cc}
\Phi^{3} & \Phi^{1}-i \Phi^{2} \\
\Phi^{1}+i \Phi^{2} & -\Phi^{3}
\end{array}\right), \\
& A_{2}=\frac{1}{2}\left(\begin{array}{cc}
0 & \Phi^{4}+i \Phi^{5} \\
-\Phi^{4}-i \Phi^{5} & 0
\end{array}\right),
\end{aligned}
$$

one can easily show that the $\mathrm{Sp}(2)$ field theory is equivalent to the $\mathrm{O}(5) \sigma$ model with Lagrangian

$$
\mathcal{L}_{\mathrm{O}}=\frac{1}{g} \partial_{\mu} \Phi \cdot \partial_{\mu} \Phi, \quad \Phi \cdot \Phi=1 .
$$

\section{CONCLUSIONS}

We have studied a 2D lattice non-Abelian gauge model with multicomponent scalar fields, focusing on the role that global and local non-Abelian gauge symmetries play in determining the universal features of the asymptotic 
low-temperature behavior. The lattice model we consider is obtained by partially gauging a maximally $\mathrm{O}(M)$-symmetric multicomponent scalar model, using the Wilson lattice approach. The resulting theory is locally invariant under $\mathrm{SU}\left(N_{c}\right)$ gauge transformations $\left(N_{c}\right.$ is the number of colors) and globally invariant under $\mathrm{SU}\left(N_{f}\right)$ transformations ( $N_{f}$ is the number of flavors). The fields belong to the coset $S^{M} / \mathrm{SU}\left(N_{c}\right)$, where $M=2 N_{f} N_{c}$ and $S^{M}$ is the $M$-dimensional sphere. The model is always disordered at finite temperature, in agreement with the Mermin-Wagner theorem [9]. However, it develops a critical behavior in the zero-temperature limit. The corresponding universal features are determined by means of numerical analyses of the FSS behavior in the zero-temperature limit.

We observe universality with respect to the inverse gauge coupling $\gamma$ that parametrizes the strength of the gauge kinetic term; see Eq. (3). The RG flow is always controlled by the infinite gauge-coupling fixed point, corresponding to $\gamma=0$, as it also occurs in three dimensions [7,8], and in 2D and $3 \mathrm{D}$ models characterized by an Abelian U(1) gauge symmetry [19,29]. Indeed, models corresponding to different values of $\gamma$ have the same universal behavior for $T \rightarrow 0$, at least in a large interval around $\gamma=0$. We conjecture that the same critical behavior is obtained for all positive finite values of $\gamma$, since, by increasing $\gamma$, we do not expect any qualitative change in the structure of the minimum-energy configurations that control the statistical average. On the other hand, the behavior for negative values of $\gamma$, i.e., when the system is frustrated, is not completely understood. Therefore, we cannot exclude that the behavior changes for large negative values of $\gamma$. This issue remains an open problem. It is important to note that by considering a positive value of $\gamma$, we are effectively investigating the behavior close to the multicritical point $\beta=\infty$ and $\beta_{g}=\beta \gamma=\infty$. Our results show that approaching the point along the lines $\beta_{g} / \beta=\gamma$ does not change the universal features of the asymptotic behavior. However, we expect that, by increasing $\beta_{g}$ faster than $\beta$ (in a well-specified way), one can observe a radical change in the critical behavior. For instance, if we take first the limit $\beta_{g} \rightarrow \infty$ at fixed finite $\beta$ and then the limit $\beta \rightarrow \infty$, the model becomes equivalent to the standard $\mathrm{O}(M)$ vector model, characterized by a different asymptotic low-temperature behavior.

The numerical results and theoretical arguments presented in this paper suggest the existence of a wide universality class characterizing 2D lattice Abelian and non-Abelian gauge models, which only depend on the global symmetry of the model. The gauge group does not apparently play any particular role. Indeed, we report numerical evidence that, for any $N_{c}$, the asymptotic lowtemperature behavior of the multiflavor scalar gauge theory (3) belongs to the universality class of the $2 \mathrm{D} \mathrm{CP}^{N_{f}-1}$ model. This also implies that it has the same universal features of the $N_{f}$-component lattice scalar electrodynamics (Abelian Higgs model) [19]. It is important to note that the global symmetry group of model (3) is $\mathrm{U}\left(N_{f}\right)$, while the global symmetry group of the $\mathrm{CP}^{N_{f}-1}$ model is $\mathrm{SU}\left(N_{f}\right)$ (we disregard here discrete subgroups), so that the global symmetry group of the two models differs by a U(1) flavor group. As we have discussed in Ref. [8], the U(1) symmetry is only apparent for $N_{f}<N_{c}$, and therefore the symmetry groups of scalar chromodynamics and of the $\mathrm{CP}^{N_{f}-1}$ model are the same for $N_{f}<N_{c}$. This U(1) symmetry is instead present for $N_{f} \geq N_{c}$. However, our numerical results indicate that the $\mathrm{U}(1)$ flavor symmetry does not play any role in model (3). The universal critical behavior is only associated with the U(1)-invariant modes that are encoded in the local bilinear operator $Q_{x}$, so that the global symmetry group that determines the asymptotic behavior is $\mathrm{SU}\left(N_{f}\right)$. Note, however, that the decoupling of the $\mathrm{U}(1)$ flavor modes may not be true in other models with the same global and local symmetries. If the U(1) modes become critical, a different critical behavior might be observed. This issue deserves further investigations.

For $N_{c}=2$, the global symmetry group changes: the action is invariant under $\operatorname{Sp}\left(N_{f}\right)$ transformations. In this case, the asymptotic low-temperature behavior is expected to be described by the $\operatorname{Sp}\left(N_{f}\right)$ continuum theory. We have numerically checked it for the two-flavor model, for which the global symmetry $\mathrm{Sp}(2) / \mathbb{Z}_{2} \simeq \mathrm{SO}(5)$.

Our results lead us to conjecture that the RG flow of the 2D multiflavor lattice scalar chromodynamics in which the fields belong to the coset $S^{M} / \mathrm{SU}\left(N_{c}\right)$, where $M=2 N_{c} N_{f}$, is asymptotically controlled by the 2D statistical field theories associated with the symmetric spaces $[10,11]$ that are invariant under $\mathrm{SU}\left(N_{f}\right)$ (for $N_{c} \geq 3$ ) or $\mathrm{Sp}\left(N_{f}\right)$ (for $N_{c}=2$ ) global transformations. These symmetry groups are the same invariance groups of scalar chromodynamics, apart from a $\mathrm{U}(1)$ flavor symmetry that is present for $N_{f} \geq N_{c}>2$, which does not play any role in determining the asymptotic behavior of the model.

This conjecture may be further extended to models with different global and local symmetry groups, for instance, to those considered in Refs. [10,30]. It would be interesting to verify whether generic non-Abelian models have an asymptotic critical behavior which is the same as that of the model defined on a symmetric space that has the same global symmetry group. This issue deserves further investigations.

\section{ACKNOWLEDGMENTS}

Numerical simulations have been performed on the CSN4 cluster of the Scientific Computing Center at INFN-PISA.

\section{APPENDIX: MINIMUM-ENERGY CONFIGURATIONS}

In this Appendix, we identify the minimum-energy configurations for the action (3), summarizing the main 
arguments reported in Ref. [8] and extending them to the $\gamma \neq 0$ case.

\section{Behavior for $\gamma=0$}

We start by considering the simplest case $\gamma=0$. The global minimum is obtained by the configurations that satisfy the maximum condition

$$
\operatorname{Re} \operatorname{Tr} \varphi_{x}^{\dagger} U_{x, \mu} \varphi_{x+\hat{\mu}}=1
$$

on each link. This condition is trivially satisfied if

$$
\varphi_{x+\hat{\mu}}=U_{x, \mu}^{\dagger} \varphi_{x},
$$

which implies $Q_{x}=Q_{x+\hat{\mu}}$, and, thus, the breaking of the $\mathrm{SU}\left(N_{f}\right) / \mathbb{Z}_{N_{c}}$ symmetry for $\beta \rightarrow \infty$.

If we apply repeatedly the relation (A2) along a plaquette, we obtain the consistency condition

$$
\varphi_{x}=\Pi_{x} \varphi_{x},
$$

where $\Pi_{x}$ is the plaquette operator (4).

For $N_{c}=2$, Eq. (A3) implies that $\Pi_{x}$ is the identity matrix. The same argument allows us to show that also all Polyakov loops can be reduced to the identity matrix, so that all gauge configurations are trivial. The energyminimum configurations can therefore be written as

$$
\varphi_{x}^{a f}=W_{x}^{a b} A^{b f}, \quad U_{x, \mu}=W_{x} W_{x+\hat{\mu}}^{\dagger},
$$

where $A^{a f}$ is a generic space-independent $2 \times N_{f}$ complex matrix satisfying $\operatorname{Tr} A^{\dagger} A=1$, and $W_{x} \in \mathrm{SU}(2)$. To verify these conclusions, we have performed simulations on a $4^{2}$ lattice for very large values of $\beta$ ( $\beta$ varies between 30 and 100). The results are reported in Table I. As expected,

$$
\operatorname{Re} \operatorname{Tr} \Pi_{x}=N_{c} .
$$

We have also computed $U$ and $\left\langle\operatorname{Tr} P_{x}^{2}\right\rangle$, confirming the predictions of Ref. [8] obtained only assuming that the configurations of minimal energy are those of the form (A4),

$$
\left\langle\operatorname{Tr} P_{x}^{2}\right\rangle \equiv \frac{N_{f}+N_{c}}{1+N_{f} N_{c}}
$$

and

$$
U=\frac{\left(1+N_{f} N_{c}\right)\left(N_{f} N_{c}+4 N_{f}^{2}+N_{f}^{3} N_{c}-6\right)}{\left(N_{f}^{2}-1\right)\left(2+N_{f} N_{c}\right)\left(3+N_{f} N_{c}\right)},
$$

with $N_{c}=2$.

For $N_{c} \geq 3$, the minimum-energy condition (A3) has several classes of different solutions. If $\Pi_{x}$ satisfies Eq. (A3), we can always write it as

$$
\Pi_{x}=V \oplus 1=\left(\begin{array}{ll}
V & 0 \\
0 & 1
\end{array}\right),
$$

where $V$ is an $\mathrm{SU}\left(N_{c}-1\right)$ matrix, modulo a gauge transformation. The corresponding configurations of the fields $\varphi_{x}$ depend on the structure of the matrix $V$. If $V$ is a generic unitary matrix which does not have unit eigenvalues, Eq. (A3) implies that the field $\varphi$ is necessarily given by

$$
\begin{aligned}
& \varphi^{a f}=0 \quad a<N_{c}, \\
& \varphi^{a f}=z^{f} \quad a=N_{c},
\end{aligned}
$$

where $z^{f}$ is a unit $N_{f}$-dimensional vector. Different $\varphi$ configurations are only possible if $V$ has some unit eigenvalues. For instance, if $V=V_{1} \oplus 1$, with $V_{1}$ belonging to the $\mathrm{SU}\left(N_{c}-2\right)$ subroup, then the $\varphi$ field configurations of the form

$$
\begin{array}{rlrl}
\varphi^{a f} & =0 & a<N_{c}-1, \\
\varphi^{a f} & =w^{f} & a & =N_{c}-1, \\
\varphi^{a f} & =z^{f} & a & =N_{c}
\end{array}
$$

( $z^{f}$ and $w^{f}$ are generic $N_{f}$-dimensional vectors) satisfy the condition (A3). To understand which type of configurations dominate, we have again resorted to numerical simulations on small lattices. The results are reported in Table I. For $\Pi_{x}$, results are consistent with

$$
\left\langle\operatorname{Re} \operatorname{Tr} \Pi_{x}\right\rangle=1
$$

This relation is consistent with Eq. (A8) only if we assume that the matrix $V$ is a randomly chosen $\mathrm{SU}\left(N_{c}-1\right)$ matrix.

TABLE I. Asymptotic values for $\beta \rightarrow \infty$ on a $4^{2}$ lattice for $\gamma=0$.

\begin{tabular}{lclllll}
\hline \hline$\left(N_{c}, N_{f}\right)$ & $\left\langle\operatorname{Re} \operatorname{Tr} \Pi_{x}\right\rangle / N_{c}$ & \multicolumn{1}{c}{$S_{g} /\left(2 N_{f}\right)$} & \multicolumn{1}{c}{$U$} & \multicolumn{1}{c}{ Eq. (A7) } & \multicolumn{1}{c}{$\left\langle\operatorname{Tr} P_{x}^{2}\right\rangle$} & Eq. (A6) \\
\hline$(2,2)$ & $1.00004(2)$ & $-1.00000(1)$ & $1.191(2)$ & $1.19048 \ldots$ & $0.800(1)$ & 0.8 \\
$(2,3)$ & $1.00000(2)$ & $-0.999994(7)$ & $1.094(1)$ & $1.09375 \ldots$ & $0.714(1)$ & $0.714286 \ldots$ \\
$(3,2)$ & $0.3421(3)$ & $-0.999998(8)$ & $1.0000000(5)$ & & $1.00002(2)$ & \\
$(3,3)$ & $0.3501(4)$ & $-1.00000(1)$ & $1.000000(2)$ & & $0.99999(2)$ & \\
$(4,2)$ & $0.2534(2)$ & $-0.999997(7)$ & $1.0000000(5)$ & & $1.00001(2)$ & \\
\hline \hline
\end{tabular}


For instance, if $V=V_{1} \oplus 1$ with a generic $V_{1} \in \mathrm{SU}\left(N_{c}-2\right)$, one would instead predict $\left\langle\operatorname{Re} \operatorname{Tr} \Pi_{x}\right\rangle=2$. This result constraints the field $\varphi$ to be of the form (A9). If this is the case, the operator $P$ takes the form $P^{f g}=\bar{z}^{f} z^{g}$ in the large- $\beta$ regime. Therefore, $P$ becomes a projector and $\operatorname{Tr} P^{2}$ is predicted to be one. Analogously, also the Binder parameter should converge to one. The numerical results reported in Table I are in perfect agreement.

\section{Behavior for $\gamma \neq 0$}

Let us now determine the minimum-energy configurations for $\gamma>0$. For $N_{c}=2$, the introduction of a positive $\gamma$ is of course irrelevant: the gauge part of the action is already minimized for $\gamma=0$. The analysis for $N_{c} \geq 3$ is instead more tricky. If we define $\beta_{g}=\beta \gamma$, fixing the value of $\gamma$ corresponds to considering a particular way of approaching the limiting point $\beta=\beta_{g}=\infty$. We will now argue that the relevant $\varphi$ configurations, that is those that dominate the ensemble average, strongly depend on how the limit is taken. Imagine that one first takes the limit $\beta_{g} \rightarrow \infty$ at fixed, finite $\beta$ and then the limit $\beta \rightarrow \infty$. In this case, the limiting configurations would have the form (A4) and $\left\langle\operatorname{Tr} P_{x}^{2}\right\rangle$ and $U$ would assume the values (A6) and (A7), respectively. On the other hand, consider the opposite approach: first, we take the limit $\beta \rightarrow \infty$ at fixed, finite $\beta_{g}$, followed by $\beta_{g} \rightarrow \infty$. For finite $\beta_{g}$, the operator $\Pi_{x}$ should have the form (A8). The matrix $V$ would not be random (it should become closer to the identity as $\beta_{g}$ increases). Nonetheless, it is not expected to have an eigenvector with an eigenvalue exactly equal to one. There, the relevant $\varphi$ configurations should always be of the form (A9). It is not possible to predict a priori what are the relevant configurations if the limit is taken keeping the ratio $\gamma=\beta_{g} / \beta$ fixed and we have therefore performed simulations on very small lattices. Results for $\gamma=1$ are reported in Table II. For $N_{f}=2$, results on a lattice with $L=4$ are definitely consistent with $U=1$ and $\left\langle\operatorname{Tr} P_{x}^{2}\right\rangle=1$. For $N_{f}=3$, we observe significantly larger size corrections. We have performed a detailed study for $N_{c}=3$. We observe that the data converge to the expected results $U=1$ and $\left\langle\operatorname{Tr} P_{x}^{2}\right\rangle=1$. $U$ converges quite fast, while the second quantity converges with the expected behavior $L^{-2}$. For $N_{f}=4$, size corrections are even larger, but the extrapolations are again consistent with the expected results. If we extrapolate the estimates of $\left\langle\operatorname{Tr} P_{x}^{2}\right\rangle$ reported in Table II assuming corrections that decay as $1 / L^{2}$, we obtain results that are consistent with one.

Let us finally discuss the case $\gamma<0$, which is much less obvious, as the system shows frustration. Indeed, if we minimize the contribution of the action that depends on the fields $\varphi$, we obtain the consistency condition (A3), which requires each plaquette to have at least one unit eigenvalue. On the other hand, minimizing the plaquette term we would expect the plaquette $\Pi_{x}$ to converge to $-I$ or $-e^{ \pm i \alpha} I$, for even or odd $N_{c}$, respectively, where $I$ is the $N_{c} \times N_{c}$ identity matrix and $\alpha=\pi / N_{c}$ [correspondingly, $\operatorname{Tr} \Pi_{x}$ would converge to $-N_{c}$ or $\left.-N_{c} \cos \left(\pi / N_{c}\right)\right]$. Therefore, one cannot simultaneously minimize all local contributions: the system is frustrated.

To understand the effective behavior along the lines $\beta_{g}=\gamma \beta$, we have again studied numerically the system for a specific value of $\gamma, \gamma=-1$. The results are reported in Table III. Per $N_{c}=2$, they show the clear presence of frustration. The plaquette is not identical to the matrix $-I$ and the minimal condition (A1) is not satisfied. Nonetheless, the estimates of the Binder parameter and of the trace of $P_{x}^{2}$ are completely consistent with the results obtained for $\gamma=0$. Even for $\gamma=-1$, the fields $\varphi$ are uniformly distributed on the $N$-dimensional sphere $\left(N=4 N_{f}\right)$.

TABLE II. Asymptotic values for $\beta \rightarrow \infty$ on a $L^{2}$ lattice for $\gamma=1$. Here $S_{\phi}$ is the part of the action (3) that depends on the $\varphi$ field (for $\gamma=0$ we have $S_{g}=S_{\phi}$ ).

\begin{tabular}{|c|c|c|c|c|c|c|c|}
\hline$\left(N_{c}, N_{f}\right)$ & $\left\langle\operatorname{Re} \operatorname{Tr} \Pi_{x}\right\rangle / N_{c}$ & $S_{\phi} /\left(2 N_{f}\right)$ & $U$ & Eq. (A7) & $\left\langle\operatorname{Tr} P_{x}^{2}\right\rangle$ & Eq. (A6) & $L$ \\
\hline$(2,2)$ & $1.000018(8)$ & $-1.000006(6)$ & $1.1905(5)$ & $1.190476 \ldots$ & $0.8000(3)$ & 0.8 & 4 \\
\hline$(2,3)$ & 0.999997 (3) & $-1.000002(3)$ & $1.0937(4)$ & $1.093750 \ldots$ & $0.7144(5)$ & $0.714286 \ldots$ & 4 \\
\hline$(3,2)$ & $1.0000(1)$ & $-1.00000(1)$ & $1.0050(3)$ & & $0.9795(8)$ & & 4 \\
\hline$(3,3)$ & $0.99997(2)$ & $-1.000003(6)$ & $1.0313(7)$ & & $0.883(3)$ & & 4 \\
\hline$(3,3)$ & $0.999999(6)$ & $-0.999997(3)$ & $1.00472(6)$ & & $0.9507(4)$ & & 6 \\
\hline$(3,3)$ & $1.000007(8)$ & $-1.000000(5)$ & $1.00100(1)$ & & $0.9746(1)$ & & 8 \\
\hline$(3,3)$ & $1.000017(7)$ & $-0.999999(2)$ & $1.00032(1)$ & & $0.98536(6)$ & & 10 \\
\hline$(3,3)$ & $1.00000(2)$ & $-0.999997(2)$ & $1.00013(1)$ & & $0.9905(1)$ & & 12 \\
\hline$(3,3)$ & $1.000015(8)$ & $-0.999999(1)$ & $1.000045(3)$ & & $0.9946(2)$ & & 15 \\
\hline$(3,4)$ & $1.0000(1)$ & $-1.000004(4)$ & $1.0520(5)$ & & $0.764(2)$ & & 4 \\
\hline$(3,4)$ & $0.99999(1)$ & $-0.999998(3)$ & $1.00460(9)$ & & $0.9499(3)$ & & 6 \\
\hline$(3,4)$ & $1.00001(1)$ & $-1.000000(2)$ & $1.00099(1)$ & & $0.9746(5)$ & & 8 \\
\hline$(3,4)$ & $1.00001(1)$ & $-1.000002(3)$ & $1.00032(1)$ & & $0.98517(7)$ & & 10 \\
\hline$(4,2)$ & $0.99998(2)$ & $-0.999998(7)$ & $1.0014(2)$ & & $0.9881(3)$ & & \\
\hline
\end{tabular}


TABLE III. Asymptotic values for $\beta \rightarrow \infty$ on a $L^{2}$ lattice for $\gamma=-1$. Here $S_{\phi}$ is the part of the action (3) that depends on the $\varphi$ field (for $\gamma=0$ we have $S_{g}=S_{\phi}$ ).

\begin{tabular}{|c|c|c|c|c|c|c|c|}
\hline$\underline{\left(N_{c}, N_{f}\right)}$ & $\left\langle\operatorname{Re} \operatorname{Tr} \Pi_{x}\right\rangle / N_{c}$ & $S_{\phi} /\left(2 N_{f}\right)$ & $U$ & Eq. (A7) & $\left\langle\operatorname{Tr} P_{x}^{2}\right\rangle$ & Eq. (A6) & $L$ \\
\hline$(2,2)$ & $-0.80901(6)$ & $-0.80898(2)$ & $1.190(2)$ & $1.190476 \ldots$ & $0.8000(5)$ & 0.8 & 4 \\
\hline$(2,2)$ & $-0.80896(4)$ & $-0.80903(2)$ & $1.1905(5)$ & $1.190476 \ldots$ & $0.7999(6)$ & 0.8 & 6 \\
\hline$(2,2)$ & $-0.80899(3)$ & $-0.809022(6)$ & $1.191(1)$ & $1.190476 \ldots$ & $0.7998(6)$ & 0.8 & 8 \\
\hline$(2,3)$ & $-0.60988(6)$ & $-0.84902(2)$ & $1.0938(5)$ & $1.093750 \ldots$ & $0.7142(6)$ & $0.714286 \ldots$ & 4 \\
\hline$(2,3)$ & $-0.60982(4)$ & $-0.849028(7)$ & $1.0937(5)$ & $1.093750 \ldots$ & $0.7142(6)$ & $0.714286 \ldots$ & 6 \\
\hline$(2,3)$ & $-0.60984(8)$ & $-0.84902(3)$ & $1.0938(2)$ & $1.093750 \ldots$ & $0.7142(4)$ & $0.714286 \ldots$ & 8 \\
\hline$(3,2)$ & $-0.33333(2)$ & $-0.999995(6)$ & $1.0000010(4)$ & & $1.00000(2)$ & & 4 \\
\hline$(3,2)$ & $-0.333326(6)$ & $-0.999998(4)$ & $1.0000005(2)$ & & $1.000000(6)$ & & 6 \\
\hline$(3,2)$ & $-0.333343(6)$ & $-0.999997(4)$ & $1.0000003(3)$ & & $1.000000(5)$ & & 8 \\
\hline$(3,3)$ & $-0.333316(8)$ & $-0.99999(2)$ & $1.000000(1)$ & & $0.99999(1)$ & & 4 \\
\hline$(3,3)$ & $-0.333338(8)$ & $-1.0000(1)$ & $1.0000003(2)$ & & $0.99998(1)$ & & 6 \\
\hline$(3,3)$ & $-0.333336(4)$ & $-0.999995(5)$ & $1.0000001(1)$ & & 1.000004 (4) & & 8 \\
\hline$(3,4)$ & $-0.33331(2)$ & $-0.999999(3)$ & $1.0000003(2)$ & & $0.999999(2)$ & & 4 \\
\hline$(3,4)$ & $-0.33332(5)$ & $-1.000000(3)$ & $1.0000001(1)$ & & $1.000006(5)$ & & 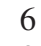 \\
\hline$(3,4)$ & $-0.33333(1)$ & $-0.999997(2)$ & $1.0000000(1)$ & & 0.999995 (4) & & 8 \\
\hline
\end{tabular}

We conclude that $\gamma$ plays a role only on the gauge properties, but not on the behavior of $\varphi$ correlations, which dominate the large- $\beta$ behavior.

For $N_{c}=3$, the results for the trace of the plaquette are completely consistent with $\Pi_{x}=\operatorname{diag}(-1,-1,1)$. In other words, the relevant gauge configurations have the same structure that holds for $\gamma=0$, Eq. (A8). The only difference is that the matrix $V$ is no longer a random $\mathrm{SU}(2)$ matrix, but is simply $-I$ (where $I$ is the two-dimensional identity). Finally, we have some results for $N_{c}=4$. In this case, it is difficult to take the limit $\beta \rightarrow \infty$, using data in the range $10 \leq \beta \leq 150$ as data still significantly change as $\beta$ increases. For $P^{2}$ and $U$, results are close to the expected ones. For a system of size $L=4$, we obtain for the average trace of $P_{x}^{2}, 0.951$ and 0.984 for $N_{f}=2$ and $\beta=50,150$, respectively (statistical errors are very small, of order $10^{-5}$ ); for $N_{f}=5$, we obtain instead 0.931 and 0.978 again for $\beta=50,150$. Again, results are consistent with a limiting value of one.
[1] S. Weinberg, The Quantum Theory of Fields (Cambridge University Press, Cambridge, United Kingdom, 2005).

[2] C. Wang, A. Nahum, M. A. Metlitski, C. Xu, and T. Senthil, Deconfined quantum critical points: Symmetries and dualities, Phys. Rev. X 7, 031051 (2017).

[3] S. Gazit, F. F. Assaad, S. Sachdev, A. Vishwanath, and C. Wang, Confinement transition of $Z_{2}$ gauge theories coupled to massless fermions: Emergent $\mathrm{QCD}_{3}$ and SO(5) symmetry, Proc. Natl. Acad. Sci. U.S.A. 115, E6987 (2018).

[4] S. Sachdev, H. D. Scammell, M. S. Scheurer, and G. Tarnopolsky, Gauge theory for the cuprates near optimal doping, Phys. Rev. B 99, 054516 (2019).

[5] H. Goldman, R. Sohal, and E. Fradkin, Landau-Ginzburg theories of non-Abelian quantum Hall states from nonAbelian bosonization, Phys. Rev. B 100, 115111 (2019).

[6] S. Sachdev, Topological order, emergent gauge fields, and fermi surface reconstruction, Rep. Prog. Phys. 82, 014001 (2019).
[7] C. Bonati, A. Pelissetto, and E. Vicari, Phase Diagram, Symmetry Breaking, and Critical Behavior of ThreeDimensional Lattice Multiflavor Scalar Chromodynamics, Phys. Rev. Lett. 123, 232002 (2019).

[8] C. Bonati, A. Pelissetto, and E. Vicari, Three-dimensional lattice multiflavor scalar chromodynamics: Interplay between global and gauge symmetries, Phys. Rev. D 101, 034505 (2020).

[9] N. D. Mermin and H. Wagner, Absence of Ferromagnetism or Antiferromagnetism in One- or Two-Dimensional Isotropic Heisenberg Models, Phys. Rev. Lett. 17, 1133 (1966).

[10] E. Brézin, S. Hikami, and J. Zinn-Justin, Generalized nonlinear $\sigma$-models with gauge invariance, Nucl. Phys. B165, 528 (1980).

[11] J. Zinn-Justin, Quantum Field Theory and Critical Phenomena, 4th ed. (Clarendon Press, Oxford, 2002).

[12] K. G. Wilson, Confinement of quarks, Phys. Rev. D 10, 2445 (1974). 
[13] H. Georgi, Weak Interactions and Modern Particle Theory (Benjamin/Cummings Publishing Company, New York, 1984).

[14] P. S. Bhupal Dev and A. Pilaftsis, Maximally symmetric two Higgs doublet model with natural standard model alignment, J. High Energy Phys. 12 (2014) 024; Erratum, J. High Energy Phys. 11 (2015) 147(E).

[15] M.E. Fisher and M. N. Barber, Scaling Theory for FiniteSize Effects in the Critical Region, Phys. Rev. Lett. 28, 1516 (1972).

[16] M. N. Barber, in Phase Transitions and Critical Phenomena, edited by C. Domb and J. L. Lebowitz (Academic Press, New York, 1983), Vol. 8.

[17] Finite Size Scaling and Numerical Simulation of Statistical Systems, edited by V. Privman (World Scientific, Singapore, 1990).

[18] A. Pelissetto and E. Vicari, Critical phenomena and renormalization group theory, Phys. Rep. 368, 549 (2002).

[19] C. Bonati, A. Pelissetto, and E. Vicari, Two-dimensional multicomponent Abelian-Higgs lattice models, Phys. Rev. D 101, 034511 (2020).

[20] S. Caracciolo and A. Pelissetto, Corrections to finite-size scaling in the lattice $N$-vector model for $N=\infty$, Phys. Rev. D 58, 105007 (1998).

[21] J. M. Kosterlitz and D. J. Thouless, Ordering, metastability and phase transitions in two-dimensional systems, J. Phys. C 6, 1181 (1973).

[22] V.L. Berezinskii, Destruction of long-range order in one-dimensional and two-dimensional systems having a continuous symmetry group I. Classical systems, Zh. Eksp. Theor. Fiz. 59, 907 (1970) [Sov. Phys. JETP 32, 493 (1971)].

[23] J. M. Kosterlitz, The critical properties of the two-dimensional xy model, J. Phys. C 7, 1046 (1974).

[24] J. V. José, L. P. Kadanoff, S. Kirkpatrick, and D. R. Nelson, Renormalization, vortices, and symmetry-breaking perturbations in the two-dimensional planar model, Phys. Rev. B 16, 1217 (1977).

[25] E. Witten, Instantons, the quark model, and the $1 / N$ expansion, Nucl. Phys. B149, 285 (1979).

[26] M. Campostrini and P. Rossi, The $1 / N$ expansion of twodimensional spin models, Riv. Nuovo Cimento 16, 1 (1993).

[27] M. Campostrini, P. Rossi, and E. Vicari, Monte Carlo simulation of $\mathrm{CP}^{N-1}$ models, Phys. Rev. D 46, 2647 (1992).

[28] An interpolation of the MC data of $U$ versus $R_{\xi}$ for the lattice $2 \mathrm{D} \mathrm{O}(5)$ vector model is provided by the function $f(y)=$ $1.40000-\left(40.78626+232.69415 y+626.04078 y^{2}\right) h(y)+$ $266.55101 y^{2}-116.42105 y^{3}-98.87100 y^{4}+73.23167 y^{5}-$ $10.81759 y^{6}$, where $h(y)=2 y\left(1-e^{-10 y}\right) /\left(1+2 e^{2 y}\right)$. This is valid for $y \in[0,0.8]$. The error is estimated to be smaller than $0.5 \%$, including the uncertainty due to the scaling corrections.

[29] A. Pelissetto and E. Vicari, Multicomponent compact Abelian Higgs models, Phys. Rev. E 100, 042134 (2019); Three-dimensional ferromagnetic $\mathrm{CP}^{N-1}$ models, Phys. Rev. E 100, 022122 (2019).

[30] A. Pelissetto, P. Rossi, and E. Vicari, Large- $N$ critical behavior of $O(M) \times O(N)$ spin models, Nucl. Phys. B607, 605 (2001). 